\title{
COMPORTAMENTO DE CATORZE PORTA-ENXERTOS PARA O LIMÃO EUREKA KM 47 NA REGIÃO DE ARARAQUARA-SP1
}

\author{
JOSÉ ORLANDO DE FIGUEIREDO ${ }^{2,4}$, JOSÉ DAGOBERTO DE NEGRI ${ }^{2}$, DIRCEU DE MATTOS JUNIOR², \\ ROSE MARY PIO ${ }^{2,4}$, FRANCISCO FERRAZ LARANJEIRA ${ }^{3,4}$, VALÉRIA XAVIER PAULA GARCIA ${ }^{2}$
}

\begin{abstract}
RESUMO - Foi avaliado o comportamento de 14 porta-enxertos para o limão 'Eureka km 47' [Citrus limon (L.) Burm. f.], clone nucelar, em experimento instalado em 1991, na Fazenda Fittipaldi, Araraquara-SP. Os porta-enxertos foram: tangelos 'Orlando' e 'Seminole' (C. reticulata Blanco x C. paradisi Macfad.), tangerinas 'Cleópatra' (C. reshni hort. ex Tanaka) e 'Sunki' [C. sunki (Hayata) hort. ex Tanaka], limões 'Cravo' (C. limonia Osbeck), 'Rugoso da África' (C. jambhiri Lush.) e 'Volkameriano Catania 2' (C. volkameriana V. Ten. \& Pasq.), laranja 'Caipira DAC' [C. sinensis (L.) Osbeck], laranja 'Azeda Doble Cálice' (C. aurantium L.), trifoliatas 'EEL'e 'Kryder 8-5' [Poncirus trifoliata (L.) Raf.], citrange 'Morton' [P. trifoliata (L.) Raf. x C. sinensis (L.) Osbeck], C. karna Raf. e C. pennivesiculata (Lush.) Tanaka. As produções foram avaliadas no período de 1998 a 2002. As maiores produções médias em peso de frutos estiveram relacionadas aos porta-enxertos C. pennivesiculata e tangerina 'Cleópatra' ( $>220 \mathrm{~kg} / \mathrm{planta)}$. Entre os porta-enxertos que induziram as mais baixas produções, estão C. karna, os dois tangelos, os dois trifoliatas e a 'Caipira DAC' (<180 kg/planta). As características de qualidade apresentadas pelos frutos e referentes ao ano de 2002 indicaram não haver diferenças expressivas devido à influência dos tratamentos. São também apresentados dados relativos à suscetibilidade das plantas à gomose de Phytophthora, no período estudado.
\end{abstract}

Termos para indexação: melhoramento, seleção, Citrus limon.

\section{BEHAVIOUR OF FOURTEEN ROOTSTOCKS FOR EUREKA LEMON [Citrus limon (L) Burm. f.] cv. km 47, in ARARAQUARA, SP, BRAZIL}

\begin{abstract}
Fourteen rootstocks were compared in a trial started in 1991, for Eureka lemon cv. km 47, nucellar clone, in Araraquara, Brazil. The rootstocks tested were Orlando and Seminole tangelos (C. reticulata Blanco x C. paradisi Macfad.), Cleopatra mandarin (C. reshni hort. ex Tanaka), Sunki mandarin [C. sunki (Hayata) hort. ex Tanaka], Rangpur Lime (C. limonia Osbeck), Rough Lemon cv. African (C. jambhiri Lush.), and Volkamerican cv Catania 2 (C. volkameriana V. Ten. \& Pasq.), sweet orange [C. sinensis (L.) Osbeck] cv. Caipira DAC, sour orange (C. aurantium L.), Poncirus trifoliata (L.) Raf. cv. EEL, P. trifoliata (L.) Raf. cv. Kryder 8-5, Morton citrange [P. trifoliata (L.) Raf. x C. sinensis (L.) Osbeck], C. karna Raf. and C. pennivesiculata (Lush.) Tanaka. Yield was measured by weight of fruits per tree, for a period of five years (1998-2002). The best yielding rootstocks were $C$. pennivesiculata and Cleopatra mandarin (> $220 \mathrm{~kg} /$ tree). Citrus karna, both tangelos, both trifoliates and sweet orange cv. Caipira DAC presented the lowest fruit yields $(<180 \mathrm{~kg} /$ tree). Fruit quality and percentage of trees affected by gummosis were also studied. No significant variation was observed for fruit quality characteristics for trees on different rootstocks, while trees on $C$. penivesiculata and sour orange were resistant to gummosis infection.
\end{abstract}

Index terms: selection, breeding, fruit yield, citrus limon.

\section{INTRODUÇÃO}

Os limões têm aceitação muito limitada entre os consumidores brasileiros no mercado interno, provavelmente por seus frutos serem consumidos quando apresentam a cor amarela e pela falta de maior conhecimento sobre suas boas qualidades. Isso se deve ao fato de que o consumo interno de frutas ácidas recai principalmente sobre a lima ácida Tahiti, erroneamente denominada de limão e cujos frutos têm coloração verde quando próprios para consumo. Acrescente-se que, quando o Tahiti adquire a cor amarela, o fruto é considerado passado. Por analogia, todo fruto amarelo de limão verdadeiro é também considerado passado.

Por outro lado, devido ao aparecimento de novas doenças às quais os limões não são sensíveis ou que apresentam baixa suscetibilidade, entre as quais a clorose variegada dos citros (CVC) e o declínio dos citros (Almeida (1995)), tem havido interesse pelo estudo dessa espécie.

Os limões são utilizados para a produção de suco concentrado congelado e para exportação como fruta fresca, ficando uma pequena porcentagem para o mercado interno. São ainda destinados para a produção de óleos essenciais e pectina, cujo valor comercial e utilização no mercado de química fina e alimentícia são bastante altos (Almeida, 1995).

O número de plantas e a área cultivada com limões no Estado de São Paulo são considerados em conjunto com as limas ácidas, tornando difícil a separação. Estima-se, porém, que existem aproximadamente 10 milhões de plantas da lima Tahiti neste Estado e que este valor representa $90 \%$ do total, restando não mais que um milhão de plantas para os limões* .

Poucos estudos têm sido feitos no Brasil objetivando a seleção de porta-enxertos para limões. A maior parte dos resultados foi obtida no exterior em outras condições edafo-climáticas (Foguet et al., 1996a, 1997; Freeman et al., 1986). O bom comportamento do clone utilizado como copa foi demonstrado em experimentos anteriores realizados no Estado de São Paulo por Figueiredo et al. (1984).

O objetivo deste trabalho foi estudar a influência de catorze porta-enxertos no comportamento do limão 'Eureka km 47، nas condições ecológicas da região de Araraquara-SP, e ampliar a base de resultados iniciais já obtidos por Figueiredo et al. (1998), por cinco $\operatorname{anos}(1998$ a 2002).

\section{MATERIALE MÉTODOS}

A instalação do experimento ocorreu em fevereiro de 1991, na fazenda Fittipaldi, município de Araraquara-SP. O espaçamento foi $8,0 \mathrm{~m}$ x 5,0 m e as plantas não receberam irrigação. A copa, limão 'Eureka km 47' e os porta-enxertos utilizados foram originários do Banco Ativo de Germoplasma de Citros do Centro APTA Citros "Sylvio Moreira", em Cordeirópolis-SP.

Os porta-enxertos foram: tangelos 'Orlando' e 'Seminole' $(C$. reticulata Blanco x $C$. paradisi Macfad.), tangerinas 'Cleópatra' ( $C$.

* M. Boteon. CEPEA, Piracicaba- SP. Comunicação pessoal, março de 2004.

\footnotetext{
${ }^{1}$ (Trabalho 134/2004). Recebido: 15/10/2004. Aceito para publicação: 19/04/2005.

${ }^{2}$ Centro APTA Citros “Sylvio Moreira”- IAC. Rod. Anhanguera, km 158. Caixa Postal 04. Cordeirópolis-SP. CEP13490-970. E-mail: joseorlando@ centrodecitricultura.br ${ }^{3}$ Embrapa-Mandioca e Fruticultura. Rua Embrapa, s/n. Caixa Postal 007. Cruz das Almas - BA. CEP 44380-000. E-mail: chico@ cnpmf.embrapa.br

${ }^{4}$ Bolsista do $\mathrm{CNPq}$
} 
reshni hort. ex Tanaka) e 'Sunki' [C. sunki (Hayata) hort. ex Tanaka], limões 'Cravo' ( $C$. limonia Osbeck), 'Rugoso da África' ( $C$. jambhiri Lush.) e 'Volkameriano Catania 2' (C. volkameriana V. Ten. \& Pasq.), laranja 'Caipira DAC' [C. sinensis (L.) Osbeck] e 'Azeda Doble Cálice' (C. aurantium L.), trifoliatas 'EEL'e 'Kryder 8-5' [Poncirus trifoliata (L.) Raf.], citrange 'Morton' [P. trifoliata (L.) Raf. x C. sinensis (L.) Osbeck], C. karna Raf. e C. pennivesiculata (Lush.) Tanaka. Foi utilizado o delineamento de blocos ao acaso, com uma planta por parcela e dez repetições, havendo somente bordaduras externas. O solo do local do experimento foi classificado como Latossolo vermelho-escuro textura média, e o clima, de acordo com a classificação de Köppen, era tipo Cwa. Os tratos culturais necessários foram os utilizados pela fazenda, sendo uniformes para todas as plantas. A produção foi obtida em todos os anos entre 1998 e 2002 e avaliada em quilos de frutos por planta, sendo as colheitas realizadas entre abril e junho.

As produções anuais de frutos para os diferentes portaenxertos estudados foram estimadas excluindo-se dados referentes a plantas severamente afetadas pela gomose de Phytophtora. A análise de variância para produção de frutos do período de estudo foi realizada tomando dados dos anos individuais como repetições e utilizandose do pacote estatístico SAS (1996). A comparação de médias considerou diferenças mínimas significativas ao nível de $P=0,05$. Em todos os anos, foi efetuada avaliação da incidência da doença gomose de Phytophthora nas plantas, fator limitante para o cultivo dos limões, por meio de observações visuais e de testes específicos de laboratório efetuados no Centro Citros "Sylvio Moreira".

As características de qualidade dos frutos foram determinadas pela avaliação da massa do fruto, teor de suco, teor de sólidos solúveis, segundo Reed et al. (1986), e teor de óleo pelo método de Scott (AOAC, 1970), coletando-se cinco amostras compostas de 15 frutos na mesma época da colheita de 2002.

\section{RESULTADOS E DISCUSSÃO}

\section{Produção}

Nesse período de 1998 a 2002, o porta-enxerto $C$. pennivesiculata proporcionou as maiores produções, tendo as plantas nele enxertadas produzido acima de $250 \mathrm{~kg} / \mathrm{planta}$, equivalente a 6,2 caixas de $40,8 \mathrm{~kg}$ de frutos, cerca de duas vezes mais que os trifoliatas, o tangelo Orlando e a laranja Caipira (Tabela1). Esse portaenxerto apresentou melhor comportamento entre aqueles estudados desde as primeiras produções após plantio no campo (Figueiredo et al., 1998). Outros estudos têm demonstrado resultados que confirmam o comportamento superior deste porta-enxerto para limões (Köller e Lichtemberg, 1984; Figueiredo et al., 1986).

A tangerina 'Cleópatra' e o 'Volkameriano' também apresentaram ótimo comportamento; entretanto, este segundo portaenxerto mostrou-se muito sensível à gomose, havendo prejuízos ao desenvolvimento da árvore ou morte, na taxa de $60 \%$ das plantas

TABELA 1 - Produções anuais e média do período de 1998 a 2002, do limão Eureka km 47, sobre catorze porta-enxertos, em Araraquara-SP.

\begin{tabular}{|c|c|c|c|c|c|c|c|}
\hline \multirow[t]{2}{*}{ Porta-enxerto } & \multicolumn{7}{|c|}{ Produção de frutos } \\
\hline & 1998 & 1999 & 2000 & 2001 & 2002 & Média $^{1}$ & $\mathrm{CV}$ \\
\hline & \multicolumn{6}{|c|}{$\mathrm{kg} /$ planta } & $\%$ \\
\hline Citrus pennivesiculata & 290 & 282 & 253 & 214 & 226 & $253 \mathrm{a}$ & 13,1 \\
\hline Tangerina 'Cleópatra' & 236 & 241 & 200 & 233 & 208 & $223 \mathrm{ab}$ & 8,2 \\
\hline Citrus volkameriana 'Catania 2' & 252 & 281 & 162 & 214 & 193 & $221 \mathrm{a}-\mathrm{c}$ & 21,4 \\
\hline Laranja 'Azeda DC' & 215 & 259 & 183 & 230 & 209 & $219 \mathrm{a}-\mathrm{d}$ & 12,7 \\
\hline Tangerina 'Sunki' & 210 & 214 & 201 & 227 & 220 & 214 a-e & 4,5 \\
\hline Limão 'Cravo' & 197 & 254 & 183 & 223 & 196 & $210 b-f$ & 13,4 \\
\hline Limão 'Rugoso da África' & 246 & 218 & 154 & 212 & 213 & $208 \mathrm{~b}-\mathrm{g}$ & 16,1 \\
\hline Citrange 'Morton' & 226 & 211 & 163 & 191 & 211 & $200 \mathrm{~b}-\mathrm{h}$ & 12,0 \\
\hline Citrus karna & 194 & 223 & 147 & 192 & 183 & $188 \mathrm{~b}-\mathrm{i}$ & 14,7 \\
\hline Tangelo 'Seminole' & 157 & 180 & 135 & 213 & 176 & $172 \mathrm{~g}-\mathrm{j}$ & 16,7 \\
\hline Trifoliata 'Kryder 8-5' & 197 & 182 & 132 & 178 & 140 & $166 \mathrm{~h}-\mathrm{k}$ & 17,1 \\
\hline Trifoliata 'EEL' & 162 & 182 & 154 & 173 & 154 & $165 \mathrm{~h}-\mathrm{k}$ & 7,5 \\
\hline Tangelo 'Orlando' & 167 & 171 & 103 & 156 & 172 & $154 \mathrm{jk}$ & 19,0 \\
\hline Laranja 'Caipira DAC' & 148 & 156 & 69 & 127 & - & $125 \mathrm{k}$ & 31,4 \\
\hline
\end{tabular}

${ }^{1}$ Diferença mínima significativa $(P=0,05)$

TABELA 2 - Incidência de gomose em plantas do limão Eureka km 47, sobre catorze porta-enxertos - dados cumulativos para o período 1998-2002. Araraquara-SP.

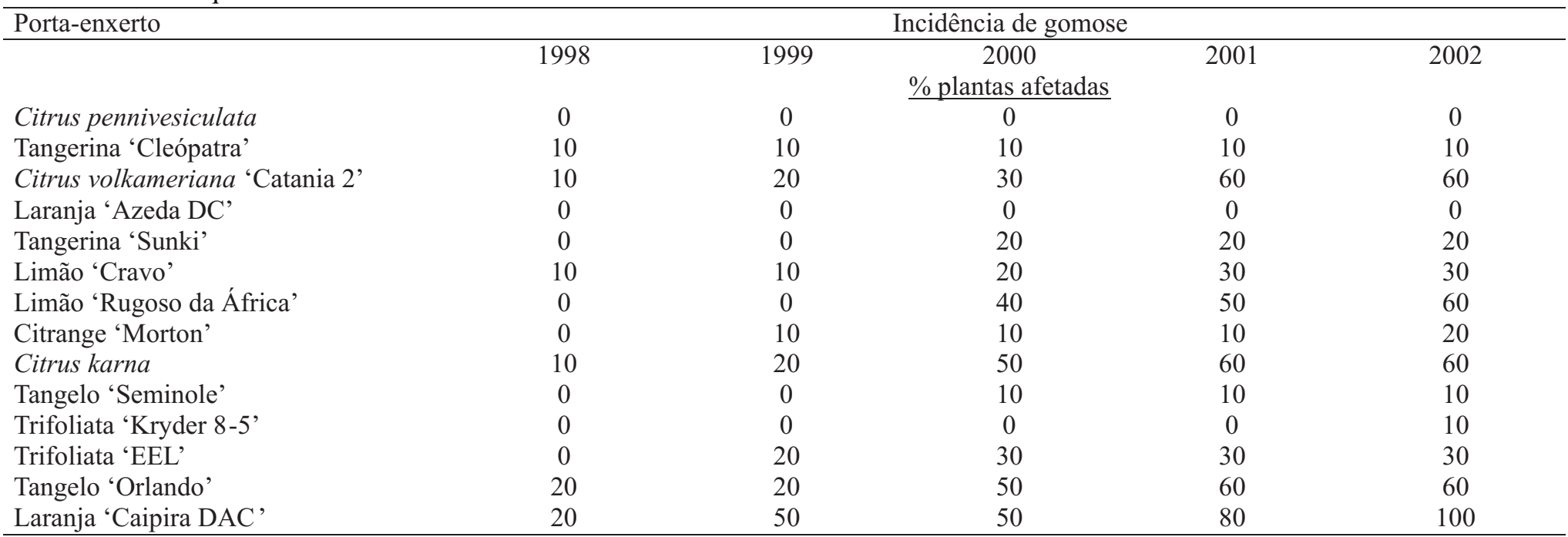


nele enxertadas ao final de onze anos de avaliação (Tabela 2).

A 'Cleópatra' proporcionou elevação significativa da produção de frutos entre os dois períodos considerados, passando de cerca de $90 \mathrm{~kg} /$ planta (Figueiredo et al., 1998) para aproximadamente $220 \mathrm{~kg} /$ planta no período de 1998 a 2002, o que denota perfeita compatibilidade entre a copa e este porta-enxerto, contrastando com as informações apresentadas por outros autores, trabalhando com porta-enxertos para limões (Dornelles, 1979; Köller, 1987). Esta tendência pode ser justificada pela característica de produção tardia de frutos de copas de citros enxertadas em Cleópatra (Pompeu Jr., 1991), o que determinaria a menor produção média do pomar nos primeiros anos após o plantio. Já o limão 'Cravo', porta-enxerto bastante utilizado para limões (Salibe e Mischan, 1981; Koller e Lichtemberg, 1984; Figueiredo et al., 1986), diminuiu a performance nos últimos anos em relação à produção, provavelmente devido à baixa resistência das plantas à gomose. Nesse tratamento, a incidência da doença e morte de plantas foi de $30 \%$ (Tabela 2).

Stenzel e Neves (2004) demonstram que a produção acumulada em oito safras da lima ácida 'Tahiti' sobre limões 'Cravo', 'Rugoso da África' e 'Volkameriano' foi cerca de $28 \%$ superior comparada aos porta-enxertos trifoliata e tangerinas 'Sunki' e 'Cleópatra'. A resposta diferencial destes dois grupos de portaenxertos corresponde àquelas observadas no presente trabalho, com exceção da tangerina 'Cleópatra', cuja menor produção de frutos foi atribuída ao menor vigor da copa de 'Tahiti'.

O porta-enxerto laranja 'Azeda', também considerado adequado para limões (Figueiredo et al., 1986), proporcionou boas produções (média de $219 \mathrm{~kg} /$ planta), que foram consideradas intermediárias neste experimento (Tabela 1). Ficou também demonstrada sua boa resistência à gomose, pois não ocorreu morte de plantas até a conclusão dos estudos (Tabela 2).

As baixas produções, em ordem decrescente, situaram-se a partir de C. karna até 'Caipira DAC' (Tabela1), o que, de certa forma, está de acordo com as tendências iniciais de produção apresentadas pelas plantas (Figueiredo et al., 1998). Os outros porta-enxertos determinaram produções intermediárias que variaram em torno de $200 \mathrm{~kg} /$ planta. O citrange 'Morton' incluiu-se nesse grupo e não repetiu seu bom comportamento obtido em outro experimento com copa de limão ‘Siciliano' (Figueiredo et al., 1986).

\section{Gomose de Phytophthora}

O acompanhamento da ocorrência de gomose nas plantas foi realizado desde seu plantio. Esses estudos revelaram que somente em $C$. pennivesiculata e em laranja 'Azeda' não houve a incidência da mesma até os 11 anos de idade das plantas, que continuavam apresentando boas condições de sanidade, não se identificando lesões causadas pela doença no tronco das árvores (Tabela 2).

Os demais porta-enxertos apresentaram porcentagens crescentes de infecção e morte de plantas no decorrer dos cinco anos estudados, e em 2002 variavam de $10 \%$ para o trifoliata 'Kryder 8-5', o tangelo 'Seminole' e a 'Cleópatra', até $100 \%$ de mortalidade das plantas sobre laranja 'Caipira DAC' (Tabela 2). A incidência de gomose nas plantas do limão mostrou alta correspondência com as caracterísitcas de cada variedade de porta-enxerto descrita por Graham e Menge (1999). Ainda, a maior severidade da doença pode ser relacionada à baixa resistência do limão à infecção (Barbasso et al., 2003). Estudos recentes sobre resistência de limas e limões a essa doença demonstraram que os limões, de modo geral, apresentam maior grau de suscetibilidade a Phytophthora parasítica que as limas ácidas (Siviero et al., 2004). Os dados da Tabela 2 podem ser usados para orientar os plantios do limão 'Eureka km 47', pois é possível estimar a taxa de incidência da doença e a necessidade de replantio dos pomares para cada combinação da copa e porta-enxertos testados, o que é uma variável de planejamento importante no controle de custos de produção.

\section{Qualidade dos frutos}

As características de qualidade dos frutos são referentes ao ano de 2002 e foram representadas por massa do fruto, teores de suco, sólidos solúveis ( ${ }^{\circ}$ Brix) e óleo. Foi observado que, com relação à massa dos frutos, os valores médios obtidos foram correspondentes à média esperada para a variedade copa, entre $144 \mathrm{~g}$ (citrange 'Morton') e $178 \mathrm{~g}$ (trifoliata 'Kryder 8-5') (Tabela 3). Frutos menores foram observados em estudos com a copa de 'Siciliano' (Donadio et al., 1995; Figueiredo, 1991). O teor de suco para todos os portaenxertos situou-se próximo a $50 \%$, valor bastante adequado se comparado aos $35 \%$, índice normalmente apresentado pelos limões (Figueiredo, 1991). Este último parâmetro variou de 47 \% (limão 'Cravo'e citrange 'Morton') a 53 \% (laranja 'Azeda'). Para os teores de sólidos solúveis e óleos essenciais, os números médios obtidos foram $9,2^{\circ}$ Brix e $0,25 \mathrm{~kg}$ de óleo por caixa de $40,8 \mathrm{~kg}$, considerando todos os porta-enxertos em conjunto.

Para o caso dos óleos essenciais, resultados contrastantes foram obtidos por Foguet (1996b), com algumas seleções de limões, entre as quais o Eureka, enxertados em 'Volkameriano' e citrange 'Troyer', mostrando haver diferenças entre os teores produzidos. Maiores variações nos teores de óleo da casca podem ser associadas à resposta do limoeiro à adubação com N, P e K (Quaggio et al., 2002). No presente estudo, os porta-enxertos não determinaram diferenças relevantes nas características de qualidade dos frutos $(P=0,05)$, cuja

TABELA 3 - Características de qualidade dos frutos do limão Eureka km 47, sobre diferentes porta-enxertos - valores médios relativos ao ano de 2002 , em Araraquara-SP.

\begin{tabular}{|c|c|c|c|c|}
\hline Porta-enxerto & Massa fruto $^{1}$ & Teor suco & Brix & Teor óleo \\
\hline & $\mathrm{g}$ & $\%$ & & $\mathrm{~kg} / \mathrm{cx}$ \\
\hline Citrus pennivesiculata & $157^{ \pm 15}$ & $50^{ \pm 1}$ & $9,0^{ \pm 0,1}$ & $0,26^{ \pm 0,03}$ \\
\hline Tangerina 'Cleópatra' & $169^{ \pm 11}$ & $50^{ \pm 1}$ & $9,0^{ \pm 0,5}$ & $0,23^{ \pm 0,01}$ \\
\hline Citrus volkameriana 'Catania 2' & $166^{ \pm 19}$ & $49^{ \pm 3}$ & $9,1^{ \pm 0,1}$ & $0,24^{ \pm 0,01}$ \\
\hline Laranja 'Azeda DC' & $162^{ \pm 9}$ & $53^{ \pm 3}$ & $9,4^{ \pm 0,3}$ & $0,27^{ \pm 0,03}$ \\
\hline Tangerina 'Sunki' & $147^{ \pm 1}$ & $51^{ \pm 2}$ & $9,3^{ \pm 0,3}$ & $0,28^{ \pm 0,03}$ \\
\hline Limão 'Cravo' & $172^{ \pm 26}$ & $47^{ \pm 4}$ & $9,3^{ \pm 0,5}$ & $0,24^{ \pm 0,05}$ \\
\hline Limão 'Rugoso da África' & $176^{ \pm 37}$ & $48^{ \pm 4}$ & $9,7^{ \pm 0,3}$ & $0,24^{ \pm 0,01}$ \\
\hline Citrange 'Morton' & $144^{ \pm 16}$ & $47^{ \pm 3}$ & $9,6^{ \pm 0,6}$ & $0,25^{ \pm 0,03}$ \\
\hline Citrus karna & $177^{ \pm 7}$ & $52^{ \pm 2}$ & $9,0^{ \pm 0,2}$ & $0,25^{ \pm 0,04}$ \\
\hline Tangelo 'Seminole' & $151^{ \pm 19}$ & $50^{ \pm 6}$ & $9,2^{ \pm 0,4}$ & $0,28^{ \pm 0,01}$ \\
\hline Trifolita 'Kryder 8-5' & $178^{ \pm 19}$ & $51^{ \pm 2}$ & $9,1^{ \pm 0,5}$ & $0,27^{ \pm 0,03}$ \\
\hline Trifoliata 'EEL' & $165^{ \pm 12}$ & $51^{ \pm 1}$ & $9,2^{ \pm 0,2}$ & $0,25^{ \pm 0,05}$ \\
\hline Tangelo 'Orlando' & $155^{ \pm 8}$ & $48^{ \pm 5}$ & $9,0^{ \pm 0,4}$ & $0,24^{ \pm 0,01}$ \\
\hline Laranja 'Caipira DAC' & $163^{ \pm 41}$ & $49^{ \pm 3}$ & $9,5^{ \pm 0,2}$ & $0,25^{ \pm 0,03}$ \\
\hline
\end{tabular}

${ }^{1}$ Valor médio ${ }^{ \pm \text {desvio padrão }}(n=5)$ 
resposta é similar àquela observada mais recentemente em experimento com lima ácida Tahiti sobre diferentes porta-enxertos (Stenzel et al., 2004).

\section{CONCLUSÕES}

O C. pennivesiculata e a tangerina 'Cleópatra' apresentaram o melhor comportamento para o limão 'Eureka km 47' nas condições ecológicas de Araraquara e semelhantes.

\section{REFERÊNCIAS}

ALMEIDA, E.L.P. Limão-Siciliano: mais uma opção para o citricultor. Laranja, Cordeirópolis, v.16, n.2, p.335-339, 1995.

AOAC - Association of Official Analytical Chemistry. Official methods of analysis. Washington: AOAC, 1970. 1015p.

BARBASSO, D.V.; SIVIERO, A.; MASUDA, Y.; BUENO Jr., J.A.S.; FIGUEIREDO, J.O. de; MACHADO, M.A. Reação de clones de limão 'Siciliano' à gomose de Phtophthora. Laranja, Cordeirópolis, v.24, n.1, p.125-134, 2003.

DONADIO, L.C.; FIGUEIREDO, J.O. de; PIO, R.M. Variedades cítricas brasileiras. Jaboticabal: FUNEP, 1995. $228 \mathrm{p}$.

DORNELLES, C.M.M. Porta-enxertos para limoeiro (Citrus limon Burmann) no Rio Grande do Sul. In: CONGRESSO BRASILEIRODE FRUTICULTURA, 5., Pelotas, 1979. Anais... Pelotas: SBF, 1979. p.2130.

FIGUEIREDO, J.O.; POMPEU Jr., J.; CAETANO, A.A.; TEÓFILO SOBRINHO, J.; IGUE, T. Melhoramento de limões verdadeiros Citrus limon Burmann, por seleção de clones. In: CONGRESSO BRASILEIRO DE FRUTICULTURA, 7., Florianópolis, 1984. Anais... Florianópolis: SBF/EMPASC,1984. p.699-709.

FIGUEIREDO, J.O.; POMPEU Jr., J.; TEÓFILO SOBRINHO, J.; REIS, A.J.; MARTINS, A.L.M.; HIROCE, R.; IGUE, T. Competição de 16 portaenxertos para o limoeiro Siciliano Citrus limon (L.) Burm. f., clone nucelar. In: CONGRESSO BRASILEIRO DE FRUTICULTURA, 8.; Brasília, 1986. Anais... Brasília: EMBRAPA-DDT/CNPq, 1986. p.133139.

FIGUEIREDO, J.O. de. Variedades copa de valor comercial. In: RODRIGUEZ, O.; VIÉGAS, F.; POMPEU JUNIOR, J. ;AMARO, A.A. (Ed.) Citricultura brasileira. 2.ed. Campinas: Fundação Cargill, 1991. v.1., p.228-264.

FIGUEIREDO, J.O. de; POMPEU Jr., J.; PIO R.M.; LARANJEIRA, F.F.; TEÓFILO SOBRINHO, J.; TANNURI, F.; FITTIPALDI, E. Produção e desenvolvimento vegetativo do limão Eureka km 47 sobre catorze porta-enxertos na região de Araraquara, SP. Revista Brasileira de Fruticultura, Cruz das Almas, v.20, n.2, p.195-201, 1998.

FOGUET, J.L.; GONZÁLES, J.L.; VINCIGUERRA, H.F.; BLANCO, A.S. Comportamiento de selecciones e hibridos de Poncirus trifoliata
(L.) Raf. como portainjertos para limonero Lisboa Frost Nuc. Revista Industrial y Agrícola de Tucuman, Tucumán, v.73, n.1-2, p.45-49, 1996a.

FOGUET, J.L.; VINCIGUERRA, H.F.; ZOSSI, S.; BLANCO, A.S.; GONZÁLES, J.L. Rendimiento y calidad del aceite esencial de 11 cultivares y 10 selecciones de limoneros (Citrus limon (L.) Burm. f.). Revista Industrial y Agrícola de Tucuman, Tucumán, v.73, n.1-2, p.51-54, 1996b.

FOGUET, J.L.; OSTE, C.A.; ALVAREZ, S.; GONZÁLES, J L.; DELFINI, A. Desarrollo, productividad y calidad de la fruta del limonero Eureka sobre nueve portainjertos. Revista Industrial y Agrícola de Tucuman, Tucumán, v.54, n.1, p.17-27, 1977.

FREEMAN, B.; BROADBENT, P.; NICHOLLS, P. Evaluation of rootstock for five lemon scions in replant ground. Australian Journal of Experimental Agriculture, Collingwood, v.26, n.6, p.751-759. 1986.

GRAHAM, J.H.; MENGE, J.A. Root diseases. In: TIMMER, L.W.; DUNCAN, L.W. (Ed.). Citrus health management. St. Paul: The American Phytopathological Society, 1999. p.126-135.

KÖLLER, O.L.; LICHTEMBERG, L.A.; Avaliação de porta-enxertos para limão 'Siciliano'em Santa Catarina. In: CONGRESSO BRASILEIRO DE FRUTICULTURA, 7., 1984, Florianópolis. Anais... Florianópolis: SBF/EMPASC, 1984. p.672-682.

KÖLLER, O.L. Comparison of rootstock for lemon 'Siciliano' (Citrus limon Burm.) in the state of Santa Catarina. Brazil. Revista Brasileira de Fruticultura, Cruz das Almas, v.9, n.1, p.37-42, 1987.

POMPEU JUNIOR, J. Porta-enxertos. In: RODRIGUEZ, O.; VIÉGAS, F.; POMPEU JUNIOR, J.; AMARO, A.A. (Ed.). Citricultura brasileira. 2.ed. Fundação Cargill: Campinas, 1991. v.1., p.265-280.

QUAGGIO, J.A.; MATTOS Jr., D.; CANTARELLA, H.; ALMEIDA, E.L.E.; CARDOSO, S.A.B.. Lemon yield and fruit quality affected by NPK fertilization. Scientia Horticulturae, Amsterdan, v.96, n.1-4, p.151$162,2002$.

REED, J.B.; HENDRIX JR., C.M.; HENDRIX, D.L. Quality control manual for citrus processing plants. Safety Harbour: Intercit, 1986. v.1, 250p.

SALIBE, A.A.; MISCHAN, M.M. Comportamento do limoeiro Feminello Santa Teresa enxertado sobre limoeiro Cravo e sobre quatro seleções de limoeiro Volkameriano In: CONGRESSO BRASILEIRO DE FRUTICULTURA, 6., 1981, Recife. Anais... Recife: SBF, 1981.p.542551.

SIVIERO, A; BARBASSO, D.V.; MASUDA, Y.; FURTADO, E.L.; FIGUEIREDO, J.O. de; CRISTOFANI, M.; MACHADO, M.A. Resistência de limas ácidas e cultivares de limões a Phytophthora parasitica. Summa Phytopathologica, Botucatu, v.30, n.2, p.204208, 2004.

STENZEL, N.M.; NEVES, C.S.V.J. Rootstocks for 'Thaiti' lime. Scientia Agricola, Piracicaba, v.61, n.2, p.151-155, 2004. 